\section{New insights into tumour invasion}

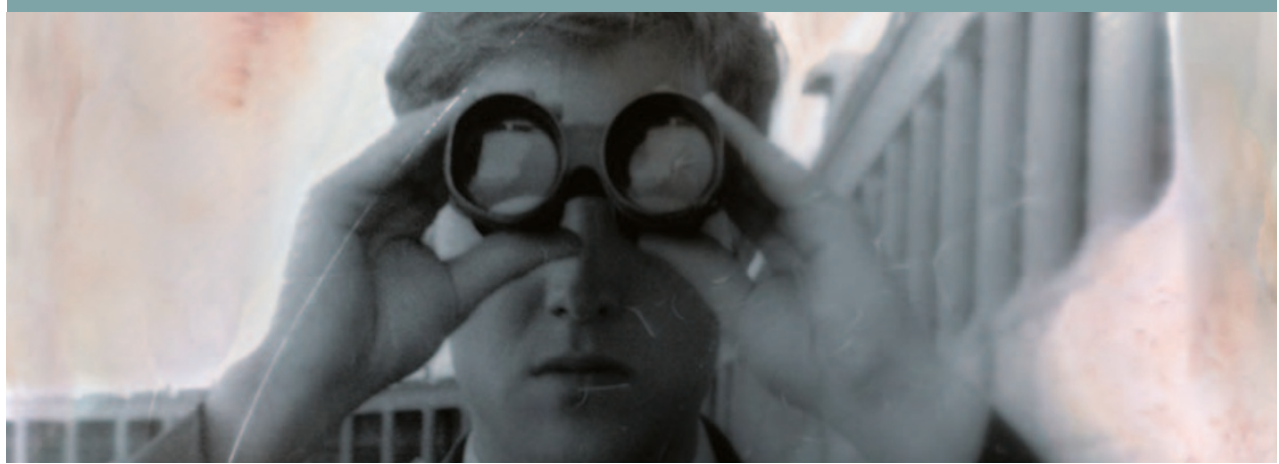

The acquisition of migratory and invasive capabilities by tumour cells is a crucial stage in the progression of solid tumours from a benign to a malignant state. Gerhard Christofori and colleagues have identified a new pathway for tumour cell invasion, driven by the small mucinlike protein podoplanin.

Tumour progression is traditionally thought to require an epithelial-mesenchymal transition (EMT) that is characterized by the loss of E cadherin expression. However, a significant number of malignant cancers do not lose E cadherin expression; furthermore, full EMT is rarely observed in biopsy samples from patients with cancer. Recently, podoplanin has been implicated in tumour progression and its expression levels are increased in cells at the invasive front of human tumours.

To investigate the functional role of podoplanin in vivo, Christofori and colleagues generated transgenic mouse lines that expressed podoplanin under the control of the rat insulin promoter, which is active in the $\beta$-cells of the pancreas (Rip1Podo mice). They then crossed Rip1Podo mice into the Rip1Tag2 transgenic mouse model of pancreatic $\beta$-cell carcinogenesis. The impact of podoplanin expression on tumour progression in the Rip1Podo;Rip1Tag2 mice was compared with single transgenic Rip1Tag2 mice. Histological analysis of pancreata from mice that were sacrificed between 12 and 13 weeks after birth showed that double-transgenic mice had a higher incidence of invasive tumours. Interestingly, no overall increase in tumour incidence (benign and invasive combined) was observed, and no other tumour-growth parameters were affected in double-transgenic mice. Furthermore, invasive carcinoma cells of double-transgenic mice did not lose $\mathrm{E}$ cadherin expression and did not upregulate other markers of EMT such as $\mathrm{N}$ cadherin.
So, how might podoplanin promote invasiveness? In tumour cells from doubletransgenic mice, a dramatic rearrangement of actin filaments was observed: subcortical actin filaments were markedly reduced and relocalized to filopodia-like foci at the plasma membrane. Could the induction of filopodia formation by podoplanin therefore affect cell migration, invasion and spreading in vitro? Expression of podoplanin in MCF7 breast many filopodia-like structures, and immunostaining showed that podoplanin is localized in these protrusions. Moreover, these cells showed increased cell spreading on extracellular matrix components and increased migratory and invasive capabilities, which indicates that their filopodia are functional.

But does podoplanin have a role in human cancers? Immunostaining of 189 human tumours (squamous-cell carcinomas and adenocarcinomas) showed that $80 \%$ of human squamous-cell carcinomas co-expressed podoplanin and E cadherin in stretches of their invasive fronts. By contrast, most adenocarcinomas did not express podoplanin. So, podoplanin induces tumour cell invasion independently of EMT by inducing filopodia formation.

Initial experiments that investigated the signalling events that underlie podoplanininduced tumour invasion indicate that podoplanin downregulates the expression of the small Rhofamily GTPases, in particular RHOA, and that this is sufficient to induce filopodia formation. Further elucidation of the molecular basis of this process might provide new targets for therapy.

Rebecca Robey, Assistant Editor, Oncogene

ORIGINAL RESEARCH PAPER Wicki, A. et al. Tumor invasion in the absence of epithelial-mesenchymal transition: podoplaninmediated remodeling of the actin cytoskeleton. Cancer Cell $\mathbf{9}$, 261-272 (2006) carcinoma cells resulted in the formation of

\section{In the news}

\section{THE RIGHT CHOICE?}

British couples undergoing IVF will now be able to have their embryos screened for hereditary breast and colon cancer.

Until now, the regulatory body, the Human Fertility and Embryology Authority (HFEA), had only allowed pre-implantation genetic diagnosis (PGD) for conditions with near-100\% penetrance and early onset, such as cystic fibrosis. In terms of cancer that meant that embryos could only be discarded on the basis of childhood retinal cancer and the colon cancer FAP. However, now they can be tested for mutations in $B R C A 1$ and BRCA2, which confer an $80 \%$ risk of breast cancer in adulthood, and mutations in the gene for hereditary non-polyposis colon cancer, which confers a similar risk. HFEA chair Suzi Leather explained: "These conditions differ from those already licensed before because people at risk do not always develop cancer, it may occur later in life and some treatments may be available." (http://www. washingtonpost.com 11 May 2006.)

The decision brought predictable criticism from 'pro-life' groups, and also from those keen to prevent the widespread use of eugenics. However, Simon Fishel, managing director of the CARE group of fertility clinics, was bullish: "If families would wish to eliminate the threat of serious cancer from their family they should be at liberty to do so." (http://www. guardian.co.uk 9 May 2006.) Richard Kennedy, of the British Fertility Society, conceded that "... the debate on the threshold of abnormality in the foetus that warrants selection at preimplantation stage still exists." (http://www.telegraph.co.uk 9 May 2006.)

Such PGD tests have been available in the United States for several years. However, because they are unregulated, no one knows the extent of their use. They are expected to be used immediately in the UK, with several patients already registered with clinics.

Patrick Goymer 